\title{
A COMPUTATIONAL TECHNIQUE TO ESTIMATE WITHIN-HOST PRODUCTIVELY INFECTED CELL LIFETIMES IN EMERGING VIRAL INFECTIONS
}

\author{
Soumya Banerjee*
}

\author{
University of Oxford \\ Oxford, United Kingdom \\ Ronin Institute \\ Montclair, United States of America \\ Complex Biological Systems Alliance \\ North Andover, United States of America
}

DOI: $10.7906 / 15.3 .3$

Regular article
Received: $17^{\text {th }}$ July 2017. Accepted: $18^{\text {th }}$ September 2017.

\begin{abstract}
Emerging viruses cause a lot of fatalities as they jump to humans from other species. Here we develop a novel technique to computationally estimate an important parameter of within-host viral infection: the lifespan of infected cells. Our approach is general and can be applied to a large class of viruses and leverages experimental data from replicon studies. Current techniques have difficulties reliably estimating infected cell lifetimes due to parameter identifiability and correlation of parameters. The infected cell lifetime is an important parameter that gives an estimate of how fast virus levels will decline. Our method would also help determine if some infected cells are short-lived or have longer lifespans with the consequence that longer lived cells could be reservoirs of infection. This would give a mechanistic understanding of why particular cell types are reservoirs of infection and may motivate therapy targeted towards these cell types. We apply our technique to West Nile virus (WNV), an emerging disease of public health relevance related to Zika virus. Our analysis suggests that the most abundant infectible cells are short-lived and could motivate therapy that targets these particular cells. Our approach is very general and can be combined with more traditional methods of using differential equation models to simulate viremia in hosts: the combination of these two techniques will likely yield results that may not be achievable using the models in isolation. This will be of great interest especially in modelling emerging diseases.
\end{abstract}

\section{KEY WORDS}

viral infections, West Nile virus, replicon studies

\section{CLASSIFICATION}

JEL: $\quad$ I19, Z19

*Corresponding author, $\eta$ : soumya.banerjee@ronininstitute.org; +1 505277 3122;

Department of Computer Scinece, 1, University of New Mexico, Albuquerque, NM, 87131, USA 


\section{INTRODUCTION}

Emerging viruses cause a lot of fatalities as they jump to humans from other species. Accurate estimates characterizing the dynamics of the effects of the virus within hosts would give insights into the disease. Here we develop a novel technique to computationally estimate an important parameter of within-host viral infection: the lifespan of infected cells. This is difficult to directly estimate based on only in-vivo (within animals) experiments. Our approach is general and can be applied to a large class of viruses and leverages experimental data from replicon studies. Mathematical models have provided important insights into within-host viral dynamics. For example, human immunodeficiency virus (HIV) infection was modeled to analyze dynamics of the virus in patients which led to estimates of the invivo rate of HIV replication and the average life span of productively infected cells [1-4]. These models gave insights into how the virus remained quiescent for years before leading to detectable symptoms. Our primary goal is to outline a novel computational technique to characterize in-vivo productively infected cell lifetimes. Our computational technique leverages advances in genetically modified viruses called replicons. Our approach can complement existing techniques using differential equations and help overcome issues of parameter identifiability and correlation of parameters [5]. The infected cell lifetime is an important parameter that gives an estimate of the how fast virus levels will decline following treatment with antivirals [4]. Accurate estimates of infected cell lifetimes would also be able to suggest if certain long lived cells could be reservoirs of infection. We motivate our analysis by applying our technique to an emerging disease: West Nile virus (WNV), which infects multiple species including humans and has caused high fatalities [6-8]. It is an arbovirus and is in the same family as other viruses such as Zika and hepatitis $\mathrm{C}$ virus which are of significant public health concern. Our analysis suggests that the most abundant infectible cells are short-lived (most likely dendritic cells and macrophages) and could motivate therapy that targets these particular cells. We suggest that our approach is very general and can be combined with more traditional methods of using differential equation models to estimate viral parameters within hosts [5]: the combination of these two techniques will likely yield results that may not be achievable using the models in isolation. This will be of great interest in modelling emerging diseases of public health significance.

\section{MATERIALS AND METHODS}

\section{STUDY DATA}

We use data on in-vivo infection of B6 mice with WNV replicons [9]. The WNV replicon was genetically engineered to encode a Renilla luciferase reporter and had the genes encoding for WNV structural proteins deleted. Viral structural proteins were packaged in trans to create virus-like particles as described in [10]. The WNV replicon was injected subcutaneously into the footpad of six week old B6 mice. At various time points, the draining popliteal lymph node was harvested, homogenized and assayed for luciferase activity.

\section{RESULTS}

\section{ANALYSIS OF DATA ON IN-VIVO INFECTION USING A WNV REPLICON}

We analyzed data on in-vivo infection of B6 mice with WNV replicons [9]. Replicons are synthetically modified viruses and in this experiment they were modified:

1) to express a gene for bioluminescence so that virus activity could be monitored, and

2) structural genes were deleted so that the replicons could not get outside the cell. 
Translation of the gene for bioluminescence produces luciferase within the infected cell which converts a substrate to photons. The activity or expression of the luciferase reporter was monitored regularly. This study has several features which help us get constraints on productively infected cell lifespans:

1) The WNV replicon kills cells in-vitro and also presumably in-vivo (personal communication Dr. Kristen Bernard, University of Wisconsin-Madison). We note however that insertion of the gene for Renilla luciferase into the WNV genome could negatively impact replication efficiency.

2) WNV replicon leads to only a single round of infection in cells.

3) The luciferase reporter is not secreted. Hence we only have to consider intracellular decay of luciferase and not clearance of luciferase outside cells (by the action of blood, extra-cellular degra- dation, etc).

We note the caveat that since the viral structural genes are deleted, there is no viral budding from infected cells and hence cytopathicity can be lower than due to the full length virus. The cells in the draining popliteal lymph node are likely dendritic cells and macrophages (personal communication, Dr. Kristen Bernard). The decline in luciferase reporter expression late in the infection is due to:

a) killing of cells in-vivo by WNV replicon leading to a decline in luciferase expression,

b) decay of luciferase within the cell,

c) migration of replicon infected dendritic cells and macrophages out of the draining lymph node and

d) inhibition of replicon replication by cellular interferon.

One study observed a small dendritic cell population in thoracic duct lymph that had presumably migrated from the lymph node and measured it to be a very small percentage (approximately $0,013 \%$ ) of all viable cells [11]. It is also not completely understood whether and how dendritic cells exit the lymph node [12]. Hence in what follows, we ignore migration of infected dendritic cells and macrophages from the draining lymph node. We represent the dynamics of luciferase activity in the declining phase by the following model:

$$
\begin{gathered}
\frac{d I_{2}}{d t}=-\delta I_{2}, \\
\frac{d L}{d t}=\kappa I_{2}-\mu L .
\end{gathered}
$$

Cells that are infected with the replicon and actively producing luciferase intracellularly are represented by $I_{2}$. Productively infected cells $\left(I_{2}\right)$ die at a rate $\delta$ per cell because of the WNV replicon, where $1 / \delta$ is the average life span of a productively infected cell. The kinetics of intracellular luciferase $(L)$ is dictated by luciferase expression within productively infected cells $\left(I_{2}\right)$ mediated by a constant $\kappa$ and intracellular decay at rate $\mu$. Solving the equations above, assuming initial conditions $L(0)=0$, leads to the following equation for the kinetics of luciferase expression within productively infected cells:

$$
L(t)=\alpha\left(e^{-\delta t}-e^{-\mu t}\right) .
$$

where $a$ is a constant. Luciferase decays rapidly within cells with a half-life of approximately 2 hours [13] yielding a decay rate $(\mu)$ of 8,3 / day. Because of this rapid decay, we can approximate the dynamics of intracellular luciferase after an initial transient phase by the following equation:

$$
L(t) \approx \alpha e^{-\delta t} \text {. }
$$

We fit Equation 4 to data in [9] (Figure 1) for the decaying phase of luciferase and estimate $\delta$ to be approximately 5.2 / day, giving us a mean productively infected lifespan $(1 / \delta)$ of approximately 5 hours $\left(r^{2}=0,85\right.$, ordinary least squares slope $\left.=-1,98\right)$. 
We also developed the complete model (without approximating that luciferase decays rapidly) and explicitly simulate the decay of luciferase. We show the results of the simulation in Figure 2. We note that it does not change the results (produces the same estimate of cell lifetimes). It predicts an initial increase in observed luciferase due to the translation of replicons to luciferase. This can be verified experimentally if it is feasible to take measurements within a few hours of inoculation by replicons.

In summary, our analysis of data from an in-vivo WNV replicon study suggests that cells in the draining lymph node, likely comprising dendritic cells and macrophages, have a productively infected cell lifespan of approximately 5 hours in mice.

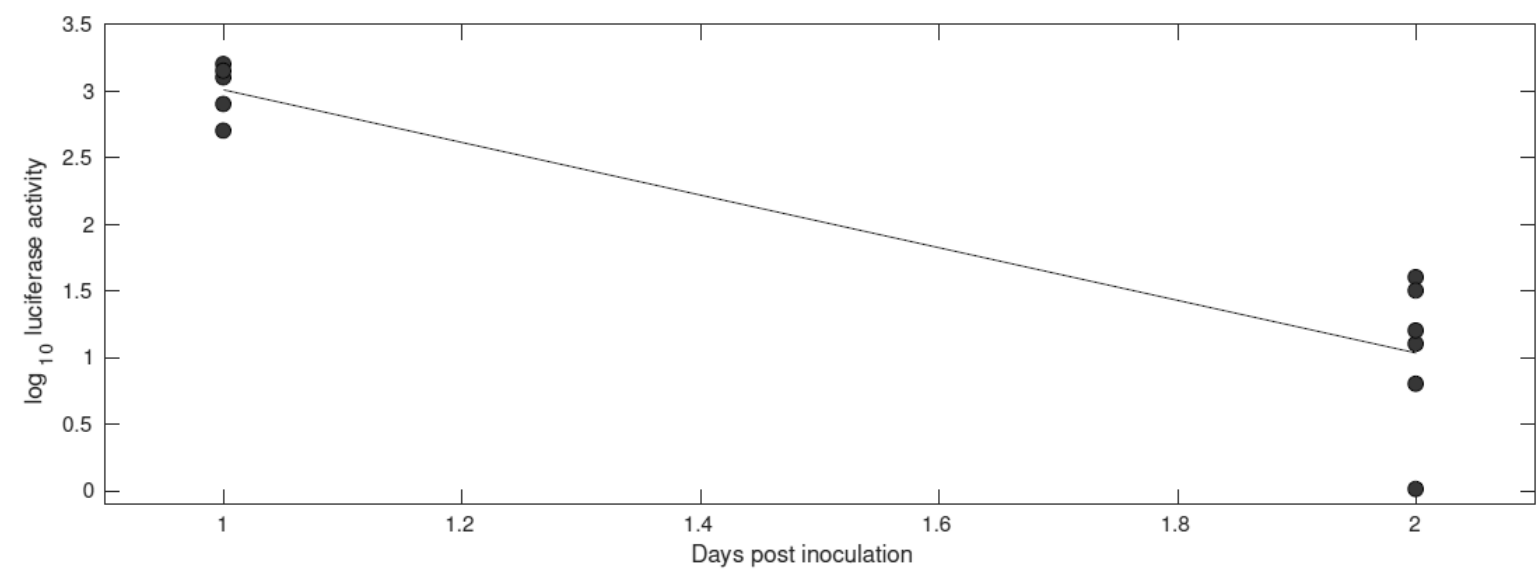

Figure 1. Equation 4 fit to data on luciferase activity in mice using a WNV replicon (data from [9]) $\left(r^{2}=0,85\right.$, ordinary least squares slope $\left.=-1,98\right)$.

\section{DISCUSSION AND CONCLUSION}

We describe a method to computationally estimate infected cell productively infected lifetimes based on experimental data for an emerging disease: West Nile virus (WNV). WNV is a flavivirus and is in the same family as other emerging diseases of public health concern such as Zika virus and hepatitis $\mathrm{C}$ virus. Our technique leverages data from a replicon system (synthetically modified virus) that encodes a gene for fluorescence which enables direct visualization of virus activity. We use a dynamical systems model to estimate an important biologically relevant parameter: the lifespan of infected cells that are producing virus. Having outlined our computational technique to infer infected cell lifetimes from a novel experimental dataset, we suggest how it can complement existing analysis of viral studies. We focus on West Nile virus (WNV) as a candidate system but a similar analysis can also be done for other viruses for which replicons can be engineered.

WNV infects multiple cell types: some are short-lived and some have longer lifespans with the consequence that longer lived cells could be reservoirs of infection. Our analysis of invivo infection data using WNV replicons (Figure 1) suggests that cells in the draining lymph node, likely comprising dendritic cells and macrophages, have a productively infected lifespan of approximately 5 hours.

A previous mathematical model trained on infection data in mice (using a non-linear differential equation model of viral kinetics) predicts a productively infected cell lifetime of 2 to 14 hours in-vivo. Our current analysis also supports this, suggesting that in-vivo lifetimes of infected cells (comprised of macrophages and dendritic cells) is approximately 5 hours. 
However, another study suggests that other cell types (keratinocytes) have longer productively infected lifespans in-vitro than our estimates in-vivo from mice [5].

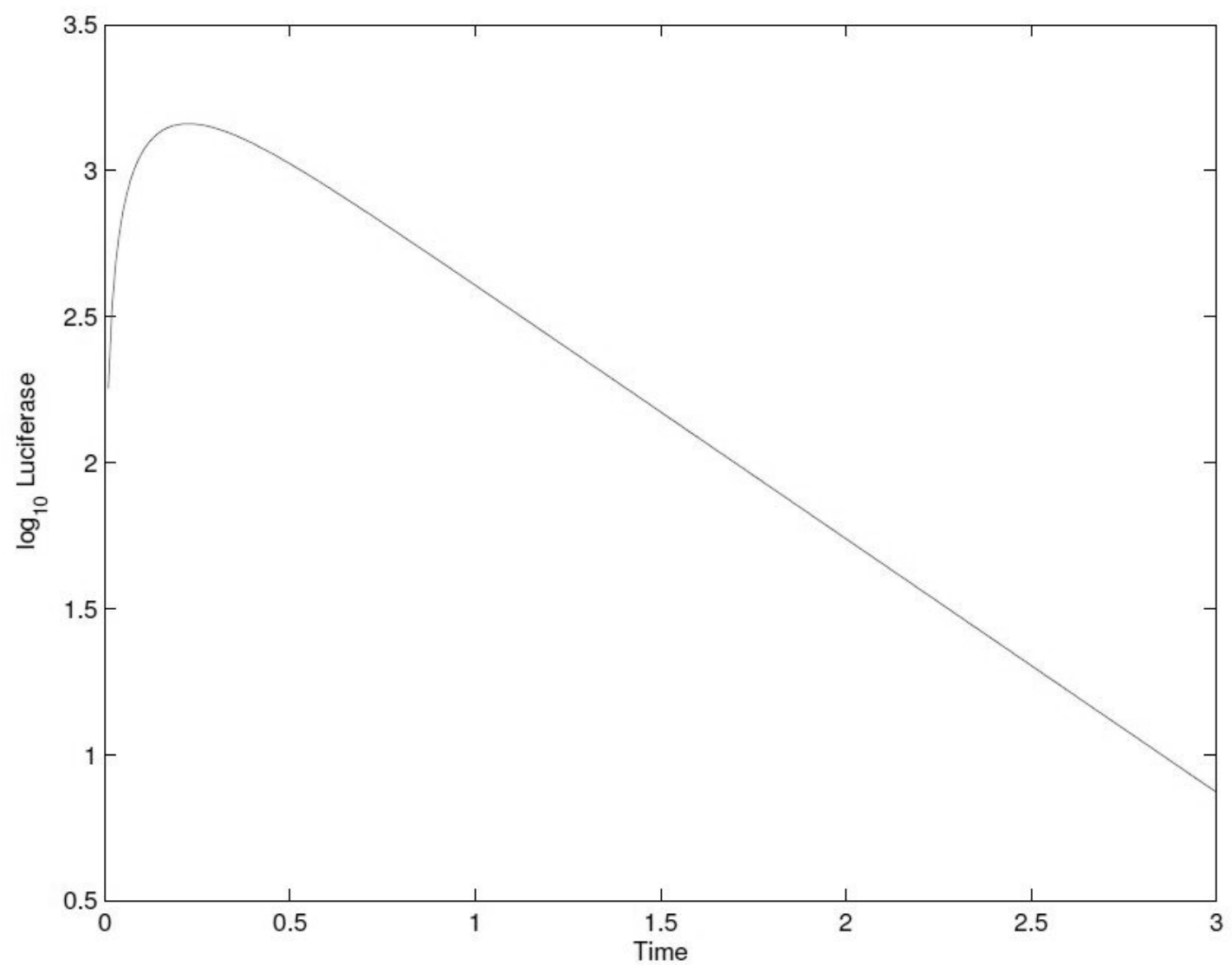

Figure 2. Simulation of complete system (Equation 3) over time (days) (explicitly modelling luciferase decay).

Is it possible to reconcile all these studies? We suggest the possibility that cells with short productively infected cell lifespans (like dendritic cells and macrophages) contribute significantly more to viremia in serum in mice than keratinocytes with longer productively infected cell lifespans. That would explain the relatively short infected cell lifetime ( 2 to 14 hours) in previous models [5] since the short-lived cells (dendritic cells and macrophages) are possibly more abundant. There are likely more susceptible dendritic cells and macrophages in the lymph node, spleen and kidney than there are keratinocytes in skin. We can reason about this as a two compartment system: one compartment is long-lived but less abundant (comprised of keratinocytes) and another compartment is short-lived but abundant (comprised of macrophages and dendritic cells). Hence the short-lived cells could contribute more to viremia and estimates of lifetimes would be closer to the lifetimes of these cells ( 2 to 14 hours).

This is akin to the situation in HIV, where infected CD4+ T-cells are short-lived and contribute significantly more to peripheral viral load than longer lived cell populations, such as macrophages [4]. Taken together with previous experimental results, our modeling suggests that cells with relatively short productively infected lifespans (likely dendritic cells and macrophages) in lymph node, spleen and kidney likely contribute significantly towards WNV viremia in serum during the acute phase of infection.

This would also be consistent with studies that inhibited replication in dendritic cells and macrophages and observed reduction of WNV titer in serum to undetectable levels [14] (also personal communication Dr. Manjunath Swamy, Texas Tech University). We note however, 
that keratinocytes also play a very important role in WNV pathogenesis. Long lived infected keratinocytes contribute towards persistent viremia in skin and peripheral organs months after infection, even well after viremia has declined to undetectable levels in serum [15]. Keratinocytes are also the initial targets of WNV infection [9] and promote migration of infected Langerhans cells to the draining lymph node [16], which in turn facilitates systemic spread of WNV [16].

In summary, our analysis suggests that the most abundant infectible cells in WNV are short-lived. These cells are most likely dendritic cells and macrophages and could be reservoirs of infection. This could motivate therapy that specifically targets these cells. Therapy during the acute phase of infection could be targeted towards short-lived cells (dendritic cells and macrophages) whereas therapy during the chronic phase can be directed towards keratinocytes. Our computational technique yields biological insights that complement approaches using differential equation models to fit viremia data [5]. This suggests a novel way to combine traditional differential equation models with models fit to replicon data and could be applied to other emerging diseases, e.g. Dengue virus for which replicons have also been constructed [17].

Finally, we note that we analyzed data from in-vivo infection of mice with WNV replicon particles modified to be capable of only one round of infection and encoding an intracellular luciferase reporter. The fact that these particles are capable of only round of infection and that they decay very rapidly in the intra-cellular environment is critical since it considerably reduces the parameters that need to be estimated.

Our modeling makes suggestions for further experimental work. More frequent measurement of replicon and simultaneous measurements with viremia in serum (both in-vivo and in the same strain of mice) may help in getting more accurate estimates of biologically relevant model parameters.

Our method is general and can be applied to other emerging viral infections where replicon experiments are feasible, e.g. in Dengue virus [17]. Our technique can complement current approaches using ordinary differential equations and can help overcome problems of parameter identifiability and correlation of parameters (like the lifetime of infected cells) [5]. The infected cell lifetime is an important parameter that gives an estimate of the how fast virus levels will decline following treatment with antivirals [4].

Accurate estimates of infected cell lifetimes can also suggest if some cell types are long-term reservoirs of infection as we have shown in the preceding analysis with WNV. This would give a mechanistic understanding of why particular cell types are reservoirs of infection and give insights into why some viruses seem to be specialized for certain cell types (cell tropism).

Accurate estimates of infected cell lifetimes can also be compared to the theoretically predicted value for optimal propagation [18] giving insights into how far a given strain of virus is from mutating into a more virulent strain. Such an analysis can also be incorporated in scaling parameters of disease progression between hosts [19]. This would enable us to couple within host models of viral kinetics to models of how viruses spread between hosts, enabling modelling of diseases at multiple scales. Our work also suggests a generality and power of dynamical models in capturing rich features of diverse complex systems ranging from immune systems and intra-cellular regulatory networks to global scientific collaboration networks [20-34].

In summary we suggest that our approach is very general and can be combined with more traditional methods of using differential equation models to estimate parameters of viremia in hosts [5]: the combination of these two techniques will likely yield results that may not be achievable using these models in isolation. This will be of great interest especially in modelling emerging diseases and viral infections of public health significance. 


\section{ACKNOWLEDGEMENTS}

Author thanks to Dr. Alan Perelson, Dr. Kristen Bernard and Dr. Manjunath Swamy for helpful discussions.

\section{REFERENCES}

[1] Ho, D.D. et al.: Rapid turnover of plasma virions and CD4 lymphocytes in HIV-1 infection.

Nature 373(6510), 123-126, 1995 , http://dx.doi.org/10.1038/373123a0,

[2] Perelson, A.S.; Kirschner, D.E. and De Boer, R.: Dynamics of HIV infection of CD4+ T cells. Mathematical Biosciences 114(1), 81-125, 1993, http://dx.doi.org/10.1016/0025-5564(93)90043-A,

[3] Perelson, A.S. et al.: HIV-1 dynamics in vivo: virion clearance rate, infected cell life-span, and viral generation time.

Science 271(5255), 1582-1586, 1996, http://dx.doi.org/10.1126/science.271.5255.1582,

[4] Perelson, A.S. et al.: Decay characteristics of HIV-1-infected compartments during combination therapy.

Nature 387(6629), 188-191, 1997, http://dx.doi.org/10.1038/387188a0,

[5] Banerjee, S. et al.: Estimating Biologically Relevant Parameters under Uncertainty for Experimental Within-Host Murine West Nile Virus Infection.

Journal of the Royal Society Interface 13(117), 2016, http://dx.doi.org/10.1098/rsif.2016.0130,

[6] Ceausu, E. et al.: Clinical manifestations in the West Nile virus outbreak.

Romanian Journal of Virology 48(1-4), 3-11, 1997,

[7] Petersen, L.R. and Marfin, A.A.: West Nile virus: a primer for the clinician. Annals of Internal Medicine 137(3), 173-179, 2002,

[8] Sejvar, J.J. et al.: Neurologic manifestations and outcome of West Nile virus infection. JAMA 290(4), 511-515, 2003, http://dx.doi.org/10.1001/jama.290.4.511,

[9] Lim, P.Y.; Behr, M.J.; Chadwick, C.M.; Shi, P.Y. and Bernard, K.A.: Keratinocytes are cell targets of West Nile virus in vivo.

Journal of Virology 85(10), 5197-5201, 2011, http://dx.doi.org/10.1128/JVI.02692-10,

[10] Puig-Basagoiti F. et al.: High-throughput assays using a luciferase-expressing replicon, virus-like particles, and full-length virus for West Nile virus drug discovery.

Antimicrobial Agents and Chemotherapy 49(12), 4980-4988, 2005, http://dx.doi.org/10.1128/AAC.49.12.4980-4988.2005,

[11] Cavanagh, L.L. et al.: Activation of bone marrow-resident memory $T$ cells by circulating, antigen-bearing dendritic cells.

Nature Immunology 6(10), 1029-1037, 2005, http://dx.doi.org/10.1038/ni1249,

[12] Alvarez, D.; Vollmann, E.H. and von Andrian, U.H.: Mechanisms and consequences of dendritic cell migration.

Immunity 29(3), 325-342, 2008, http://dx.doi.org/10.1016/j.immuni.2008.08.006,

[13] Ignowski, J.M. and Schaffer, D.V.: Kinetic analysis and modeling of firefly luciferase as a quantitative reporter gene in live mammalian cells.

Biotechnology and Bioengineering 86(7), 827-834, 2004, http://dx.doi.org/10.1002/bit.20059, 
[14] Ye, C.; Abraham, S.; Wu, H.; Shanka, P.; and Manjunath, N.: Silencing early viral replication in macrophages and dendritic cells effectively suppresses flavivirus encephalitis.

PloSOne 6(3), e17889, 2011, http://dx.doi.org/10.1371/journal.pone.0017889,

[15] Appler, K.K. et al.: Persistence of west nile virus in the central nervous system and periphery of mice.

PLoSOne 5(5), e10649, 2010, http://dx.doi.org/10.1371/journal.pone.0010649,

[16] Welte, T. et al.: Toll-like receptor 7-induced immune response to cutaneous West Nile virus infection.

Journal of General Virology 90(pt11), 2660-2668, 2011, http://dx.doi.org/10.1099/vir.0.011783-0,

[17] Yang, C.C. et al.: Characterization of an efficientdengue virus replicon for development of assays of discovery of small molecules against dengue virus.

Antiviral Research 98(2), 228-241, 2013, http://dx.doi.org/10.1016/j.antiviral.2013.03.001,

[18] Banerjee, S.: Optimal Strategies for Virus Propagation. preprint arXiv:1512.00844 [q-bio.CB], 2015,

[19]Banerjee, S.: Scaling in the Immune System. Ph.D. Thesis. University of New Mexico, Albuquerque, 2013,

[20] Banerjee, S. and Moses, M.: Scale invariance of immune system response rates and times: perspectives on immune system architecture and implications for artificial immune systems.

Swarm Intelligence 4(4), 301-318, 2010, http://dx.doi.org/10.1007/s11721-010-0048-2,

[21] Levin, D. et al.: A spatial model of the efficiency of T cell search in the influenza-infected lung. Journal of Theoretical Biology 398, 52-63, 2016, http://dx.doi.org/10.1016/j.jtbi.2016.02.022,

[22] Banerjee, S.: Analysis of a Planetary Scale Scientific Collaboration Dataset Reveals Novel Patterns. preprint arXiv:1509.07313 [cs.SI], 2015,

[23]Banerjee, S.: An Immune System Inspired Approach to Automated Program Verification. preprint arXiv:0905.2649 [cs.NE], 2009,

[24]Banerjee, S. and Moses, M.: Immune System Inspired Strategies for Distributed Systems. preprint arXiv:1008.2799 [cs.DC], 2010,

[25] Banerjee, S.; Levin, D.; Moses, M.; Koster, F. and Forrest, S.: The value of inflammatory signals in adaptive immune responses.

In: Lio, P.; Nicosia, G. and Stibor, T., eds.: Artificial Immune Systems. $10^{\text {th }}$ International Conference. Springer, Berlin \& Heidelberg, 2011, http://dx.doi.org/10.1007/978-3-642-22371-6,

[26]Banerjee, S.: A Stage Structured Hybrid Model for Disease Dynamics Modelling. The 2nd Annual Cybersecurity Early Careers Researchers Symposium, University of Oxford, Oxford, 2016, http://dx.doi.org/10.13140/RG.2.2.13054.79683,

[27]Banerjee, S. and Hecker, J.: A Multi-Agent System Approach to Load-Balancing and Resource Allocation for Distributed Computing. Complex Systems Digital Campus 2015, World e-Conference, Conference on Complex Systems, 2015,

[28]Moses, M. and Banerjee, S.: Biologically Inspired Design Principles for Scalable, Robust, Adaptive, Decentralized Search and Automated Response (RADAR). Proceedings of the 2011 IEEE Conference on Artificial Life, pp.30-37, 2011, http://dx.doi.org/10.1109/ALIFE.2011.5954663, 
[29]Banerjee, S. and Moses, M.: A hybrid agent based and differential equation model of body size effects on pathogen replication and immune system response.

In: Andrews, P.S. et al.: Artificial Immune Systems. 8th International Conference, ICARIS, Lecture Notes in Computer Science. Springer Verlag, Berlin, 2009,

[30] Banerjee, S.; van Hentenryck, P. and Cebrian, M.: Competitive dynamics between criminals and law enforcement explains the super-linear scaling of crime in cities.

Palgrave Communications 1, No. 15022, 2015, http://dx.doi.org/10.1057/palcomms.2015.22,

[31]Liu, P. et al.: A Bioorthogonal Small-Molecule- Switch System for Controlling Protein Function in Live Cells.

Angewandte Chemie - International Edition 53(38), 2014, http://dx.doi.org/10.1002/anie.201403463,

[32]Balch, C. et al.: Science and technology consortia in U.S. biomedical research: a paradigm shift in response to unsustainable academic growth.

BioEssays 37(2), 119-122, 2015,

http://dx.doi.org/10.1002/bies.201400167,

[33] Banerjee, S.: A Biologically Inspired Model of Distributed Online Communication Supporting Efficient Search and Diffusion of Innovation. Interdisciplinary Description of Complex Systems 14(1), 10-22, 2016, http://dx.doi.org/10.7906/indecs.14.1.2,

[34] Banerjee, S.: A Roadmap for a Computational Theory of the Value of Information in Origin of Life Questions.

Interdisciplinary Description of Complex Systems 14(3), 314-321, 2016, http://dx.doi.org/10.7906/indecs.14.3.4. 